Dos s i e r

$>$ La fiscalité locale regards comparatifs Colloque de Lyon

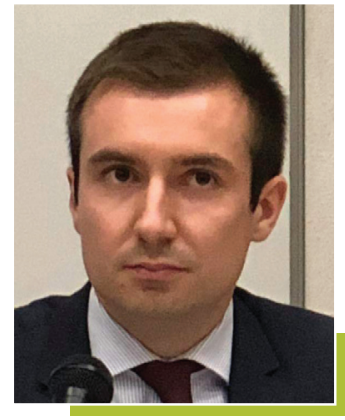

\title{
Les économies numériques et collaboratives, des opportunités nouvelles de refonte de la fiscalité locale?
}

Mots-clés : finances locales - fiscalité du numérique - économie collaborative

Si le développement des économies numériques et collaboratives est largement ignoré par la fiscalité locale, les collectivités disposent toutefois d'atouts non négligeables issus de la nature même de ces activités et peuvent en outre bénéficier de la modernisation du prélèvement fiscal à travers la collaboration des plateformes tierces. Pourtant, faute d'une réelle autonomie fiscale, le bénéfice qu'elles peuvent en tirer demeure minime.

ace au développement des économies numériques et collaboratives, la fiscalité locale peine à s'adapter. Les raisons de son inadaptation sont particulièrement nombreuses : relevons le fait, d'une part, que la fiscalité locale demeure encore largement assise sur une forme de conception «physiocratique » de l'économie, justifiant ainsi une imposition essentiellement fondée sur la propriété immobilière ; d'autre part, le développement de l'économie numérique a davantage été appréhendé au niveau des États, et au-delà, à plus forte raison en France où l'autonomie financière des collectivités territoriales est quasiment inexistante. II ne s'agit pas ici d'interroger les conceptions profondes, I'infrastructure économique, du système d'imposition; en revanche, il s'agit d'entreprendre une approche plus pratique sur les mutations du modèle économique Français afin d'établir des schémas opératoires dans lesquels les collectivités locales 
o s s i e r

$>$ La fiscalité locale : regards comparatifs Colloque de Lyon 10 octobre 2019 trouveront peut-être de quoi se démarquer et assurer ainsi leur pérennité financière pour les années avenir.

Le développement du numérique a permis l'émergence des économies numériques et collaboratives, sans pour autant constituer une rupture avec le système marchand traditionnel ${ }^{1}$. L'économie collaborative désigne les modèles économiques dans lesquels des "plateformes", généralement numériques, créent un marché ouvert pour l'utilisation temporaire de biens et de services, souvent produits ou fournis par des personnes privées, en vue de faciliter des activités économiques ${ }^{2}$. De manière plus concrète, l'économie collaborative repose sur le partage ou l'échange de biens, de services ou de connaissances, ayant ou non un caractère lucratif, par I'intermédiaire généralement d'une plateforme numérique de mise en relation. Le numérique a permis aux initiatives collaboratives de changer d'échelle et de faciliter ainsi les contacts entre membres de communautés plus large, mais il faut toujours garder à l'esprit cette distinction conceptuelle entre ce qu'est l'économie numérique et ce qu'est l'économie collaborative. Dès lors, cette dernière peut-être un embranchement de la première, notamment lorsqu'il s'agit de sa forme viciée, c'est-à-dire l' "ubérisation».

Disons dès à présent que le développement de l'économie numérique - et à plus forte raison de l'économie collaborative -, est largement ignoré par la fiscalité locale contemporaine. Ce n'est pas l'aveu d'un désintérêt de la puissance publique à l'égard d'un sujet hautement sensible et complexe, mais davantage l'incertitude de pouvoir résoudre des difficultés qui sont déjà difficilement approchées aux échelles nationale et internationale. Par ailleurs, rares sont les rapports établis par les différents acteurs de la gouvernance fiscale dans lesquels ils identifient clairement les enjeux fiscaux du développement de l'économie numérique pour les collectivités territoriales. On relève notamment, en 2014, un rapport du Conseil des prélèvements obligatoires à propos de «La fiscalité locale et les entreprises »; en revanche, le plus récent rapport dit « Richard-Bur», en date de mai 2018, sur « La refonte de la fiscalité locale » ne fait aucune mention des incidences des économies numériques ou collaboratives sur la fiscalité locale. Pour tout dire, un seul rapport, rédigé par l'Inspection Générale des Finances et remis à la fin de l'année 2018, traite longuement de ces difficultés; malheureusement et « étonnement », il n'a jamais été publié.

Bien que la fiscalité locale ignore largement le développement des économies numériques et collaboratives, nous pensons que sur un plan théorique, elle n'est pas pour autant dénuée d'atouts précieux afin de voir son rôle se renforcer. Finalement, il convient de s'interroger sur l'existence même d'éventuels bénéfices que la fiscalité locale peut tirer du développement massif des économies numériques et collaboratives.

A priori, il n'est pas possible d'identifier en quoi la fiscalité locale pourrait bénéficier du développement ces économies numériques et collaboratives; au contraire, nous ne pouvons que constater que les collectivités sont défaillantes dans l'appréhension de ce nouveau phénomène, autant parce qu'elles ne savent pas comment ces nouvelles économies affectent leurs finances qu'en raison d'un manque de compétences sur les impositions qui auraient pu leur permettre d'agir (I). Cette défaillance pourrait toutefois ne pas être insurmontable dès lors qu'elles disposent de quelques atouts dans la perspective d'une adaptation de la fiscalité directe locale aux économies numériques (II).
V. Rapport d'information fait au nom de la commission des finances sur l'économie collaborative, Propositions pour une fiscalité simple, juste et efficace, Sénat, session extraordinaire 2014-2015, Rapport n 690, 17 septembre 2015, p. 7 ; Douet Frédéric Fiscalité 2.0. Fiscalité du numérique, Paris, Lexis Nexis coll. «Précis », 2018, p. 3.

${ }^{2}$ Définition proposée par la Commission Européenne: Communication de la commission au Parlement européen, au Conseil, au Comité économique et socia européen et au Comité des régions, Un agenda Européen pour l'économie collaborative, COM(2016) 356 final, 2 juin 2016.

\section{La défaillance des collectivités territoriales dans I'appréhension fiscale du développement des économies numériques et collaboratives}

Dans la plupart des cas, la fiscalité locale ignore les questions relatives au développement de l'économie numérique et collaborative; les collectivités ne sont dès lors pas en mesure d'identifier les préjudices fiscaux qu'elles subissent (A). En outre, même lorsqu'elles sont conscientes des difficultés, elles peuvent rarement s'adapter et ainsi lutter contre les phénomènes à l'œuvre (B).

\section{A. Un phénomène économique concurrentiel encore largement ignoré}

À I'heure où l'on discute encore de la couverture numérique de l'ensemble du territoire français, les conséquences économiques de ce développement pour les collectivités territoriales sont 
largement inconnues. En premier lieu, si le numérique permet la création d'activités économiques pour les collectivités, toutes n'en bénéficient pas de la même manière ${ }^{3}$. En second lieu, les entreprises traditionnelles installées au cœur des territoires - et généralement « non connectées »-, sont fortement affectées par plusieurs phénomènes de concurrences puisque les entreprises des économies numériques et collaboratives ont la capacité de pratiquer des tarifs nettement inférieurs aux leurs. Enfin, la multiplication des impositions locales a tendance à accroître l'intensité concurrentielle locale, dès lors que ces impositions constituent un critère d'installation pour les nouvelles entreprises. Un rapport du Conseil des prélèvements obligatoires ${ }^{4}$, qui proposait une comparaison européenne des structures fiscales locales en Europe, souligne que la prolifération des taxes locales en France se traduit par un surcroît de complexité pour les entreprises : en effet, cette fiscalité est fragmentée en près de soixante-dix impositions, dont la principale est la contribution économique territoriale, créée en 2010 en remplacement de la taxe professionnelle. Outre la diversité des impositions concernées, la complexité de cette fiscalité est renforcée par son absence de définition partagée et l'éclatement des compétences relatives à son élaboration et son suivi.

Pour résister, les commerces traditionnels des centres-villes doivent adapter leur offre. Ils développent leur accès aux plateformes numériques afin d'élargir leur zone de chalandise ; parallèlement, on constate un phénomène économique nouveau avec la doctrine du localisme, permettant de lier attrait du numérique et proximité. Rappelons que pour l'heure, seules $11 \%$ des entreprises françaises vendent en ligne tandis que 59 \% des consommateurs en France ont recours aux achats sur internet ${ }^{5}$. Ce phénomène conduit à une fragilisation du tissu économique local et, indirectement, du potentiel fiscal de ces collectivités ${ }^{6}$. D'autant qu'en se développant souvent en marge de l'économie traditionnelle et, a fortiori, en marge du système fiscal, ce phénomène met en péril des pans entiers du droit et de l'économie ; ainsi, nous constatons que si des normes fiscales existent, elles sont souvent désordonnées et ne peuvent pas répondre aux besoins de réglementation fiscale au niveau local.

En juin 2018, était attendu un rapport de I'Inspection Générale des Finances, qui planchait sur toute une série de propositions fiscales en ce qui concerne le secteur du commerce, afin notamment de le rendre plus équitable avec les commerces traditionnels, notamment ceux qui peuplent les centres-villes et dont on connaît les difficultés croissantes. L'une des principales ambitions du gouvernement était de proposer une importante réforme de la taxe sur les surfaces commerciales, dont le produit est supérieur à un milliard d'euros et à laquelle échappent largement les e-marchands. Ce rapport n'a jamais été officiellement publié ; contrairement à celui édité en 2015 par le Pôle interministériel de Prospective et d'Anticipation des Mutations (PIPAM) à propos des «Enjeux et perception de la consommation collaborative $»^{7}$ duquel ressort $d^{\prime}$ importantes perspectives budgétaires, notamment à travers la possibilité pour les services publics de " générer des économies en intégrant l'économie collaborative » à leur fonctionnement. Ce rapport ignore largement les problématiques fiscales, sûrement en raison d'ailleurs des faibles marges de manœurres dont disposent les collectivités en la matière.

\section{B. Une compétence fiscale locale ina- daptée aux nouveaux enjeux écono- miques}

L'une des principales problématiques posées par les économies numériques porte sur l'évitement fiscal dont les plateformes, et leurs utilisateurs, sont à l'origine. On comprend aisément que les autorités sont généralement impuissantes, à plus forte raison les autorités locales qui ne peuvent pas, par elles même, mettre en œuvre une fiscalité dédiée à des acteurs économiques qui échappent déjà largement aux maigres dispositifs fiscaux existants. Effectivement, les collectivités ne disposent pas d'un pouvoir d'assiette étendu et ne peuvent donc ni prétendre à une éventuelle taxation, dans l'hypothèse où l'assiette préexiste, ni à l'établissement d'une telle assiette, dans l'hypothèse où il conviendrait de tout instaurer. Pour ce qui concerne l'assujettissement à la fiscalité locale actuelle, face aux risques de fracture entre les commerces traditionnels, plus particulièrement les TPE/PME, et les entreprises du numérique, notamment les "pure-players ${ }^{8}$, le Gouvernement est depuis 2018 régulièrement interpellé par les parlementaires. Certaines de ces questions donnent lieu à une réponse officielle - dont le contenu est semblable-, sans pour autant parvenir à convaincre.

Les « pures-players » profitent de leur statut de vendeur à distance, installés dans des États membres de l'Union européenne pratiquant le dumping fiscal, pour payer peu, voire aucun impôt, dans l'État où les ventes sont réalisées. $\mathrm{Si}$ le Gouvernement et le commissaire européen aux affaires économiques et financières

\footnotetext{
${ }^{3}$ Attour Amel et Longhi

Christian, "Fracture numérique, le chaînon manquant. Les services de d'eadministration locale dans les commune françaises ", in Les Cahiers du numérique, vol. 5 , 2009, pp. 119-146.

${ }^{4}$ Conseil des prélèvements obligatoires, Fiscalité locale et entreprises, Rapport mai 2014. ${ }^{5}$ Ibid, p. 58

${ }^{6}$ Inspection générale des finances, La revitalisation commerciale des centres-villes, Rapport n² 2016-M-022, juillet 2016.

${ }^{7}$ PIPAM, Enjeux et perspectives de la consommation collaborative, Rapport 2015.

${ }^{8}$ || s'agit d'une personne physique ou morale exerçant son activité exclusivement sur ou à travers l'internet.
} 
o s s i e r

$>$ La fiscalité locale : regards comparatifs Colloque de Lyon 10 octobre 2019 à la fiscalité et à l'union douanière se sont déjà engagés dans la définition d'une nouvelle « taxe d'égalisation » pour les e-commerçants afin de compenser le paiement de I'IS, il semblerait que la question de l'harmonisation de la fiscalité locale n'ait pas été abordée. Or, la dématérialisation d'activités commerciales par des acteurs essentiellement numériques tend à remettre en cause le système fiscal d'imposition local assis sur l'emprise foncière des magasins. Aujourd'hui la fiscalité locale est supportée dans sa totalité par les magasins physiques, alors même que les acteurs numériques profitent eux aussi des infrastructures locales, destinées à l'entretien des routes, à la collecte des ordures, au dépôt dans des points de vente physiques, etc. Autrement dit, les commerçants physiques se retrouvent à contribuer aux financements de l'environnement nécessaire au commerce de leurs concurrents en ligne. Concrètement, les commerces physiques sont assujettis à de nombreux impôts locaux, principalement liés à leurs investissements immobiliers auxquels ne sont pas souvent soumis les commerces numériques : cotisation financière des entreprises (CFE), taxe foncière sur les propriétés bâties, taxe locale sur les enseignes et les publicités extérieures et surtout taxe sur les surfaces commerciales. Sur le plan de la cotisation foncière des entreprises par exemple, elle frappe toutes les personnes exerçant en France une activité professionnelle non salariée qui n'entre pas dans l'un des cas d'exonération de plein droit ou facultative. Or, une personne n'entre pas dans le champ de la cotisation foncière des entreprises dès lors qu'elle n'est assujettie ni à l'impôt sur le revenu ni à l'impôt sur les sociétés en raison des règles de territorialité applicables à ces impôts. $C^{\prime}$ est notamment le cas des grandes entreprises du numérique, ainsi exemptées de CFE alors même qu'elles exercent une activité entrant dans le champ d'application de cet impôt (art. 1447, I du CGI). Les budgets des collectivités locales sont évidemment affectés par ce phénomène, sans même envisager les externalités fiscales négatives en termes de dépeuplement des territoires ou de délaissement des centres-villes par les entreprises. Lorsque le gouvernement répond', souvent il n'envisage pas le sort collectivités et, lorsqu'il le fait, c'est généralement pour renvoyer à un rapport non publié ou simplement pour rappeler les travaux internationaux en cours.

Les impôts locaux sont donc parfaitement inadaptés à ces phénomènes nouveaux de concurrence, tant en raison de leur nature qu'en raison d'un désintéressement de la plupart des collectivités, quand bien même elles disposent d'atouts non négligeables, dès lors qu'elles préfèrent légitimement s'assurer la perception de leur dotation de la part de l'État que de se lancer dans des entreprises fiscales aventureuses, qui nécessiteraient une autonomie juridique.

\section{Le renouvellement des perspectives fiscales des collectivités territoriales par le développement des économies numériques et collaboratives}

Les collectivités territoriales ne sauraient demeurer passives face au développement des économies numériques et collaboratives. Au contraire, elles disposent a priori d'outils efficaces pour déterminer et profiter des bases imposables de ces nouvelles formes d'économies (A), d'autant qu'elles peuvent aussi bénéficier de l'appui des tiers et de la modernisation du recouvrement subséquente (B).

\section{A. Une nouvelle détermination des bases imposables favorables à l'éche- lon local}

Si l'économie numérique se fonde essentiellement sur des transactions numériques, elle n'en est pas moins incarnée physiquement puisqu'il est possible de discerner un flux économique à destination ou entre des personnes qui, elles, ont une existence physique territoriale. Il est particulièrement difficile pour les collectivités d'atteindre ces flux économiques nouveaux dès lors qu'elles ne maîtrisent pas les outils nécessaires en termes de détermination des bases imposables. Effectivement, la fiscalité locale demeure pleinement une fiscalité d'attribution et de redistribution; d'ailleurs, la majorité des prélèvements locaux sont décidés et exécutés par l'État et sa toute puissante administration. Les exceptions sont rares : en ce qui concerne la location immobilière meublée par exemple, les collectivités n'hésitent pas à employer du personnel municipal pour arpenter l'internet et les rues à la recherche des fraudeurs - parfois involontaires.

Les collectivités bénéficient toutefois de la connaissance du terrain comme atout et, parfois même, elles participent à l'émergence de 
nouvelles activités qu'elles identifient donc plus rapidement que l'État, y compris lorsqu'elles relèvent, dans les premiers temps de leur existence, de l'économie informelle. Il en est ainsi des logements mis en location, mais il en est aussi du développement des marchés parallèles (localisme, AMAP, SEL, etc.). À moyen terme, les externalités fiscales provenant de ces économies profitent à tous.

Bien conscient de l'implication dans les territoires de ces nouvelles économies, le Comité européen des régions ${ }^{10}$, dans un avis publié en 2017, a entendu penser un cadre réglementaire commun d'orientation et de pilotage. Le Comité constate que l'impact territorial des économies numériques est important et, selon lui, la dimension locale et régionale du phénomène collaboratif participe pleinement à l'essor de ce dernier. Pour autant, le même Comité remarque avec justesse le manque d'association des collectivités locales et régionales à ces problématiques : pour cause, les États tendent - tendaient - à se saisir seuls des questions liées au développement de cette économie, notamment sur le plan social ou fiscal (tel est le cas en Belgique ou en Italie). Plus spécifiquement, le Comité estime que des politiques publiques adéquates relativement à ces nouvelles économies peuvent aussi participer à la réduction des dépenses publiques. En ce sens, notons que le rapport «Terrasse » proposait de promouvoir des territoires collaboratifs expérimentaux ${ }^{11}$. L'objectif était d'impliquer les collectivités territoriales dans une politique qui était censée soutenir les expérimentations innovantes d'économie collaborative, en particulier dans les territoires ruraux qui en ont besoin. Cette politique s'inscrit dans le cadre de la lutte contre le délaissement des territoires ruraux et des centres-villes de petites et moyennes villes. En ce sens, nous pensons qu'elle pourrait prendre la forme de dispositifs fiscaux incitatifs, pleinement tournés vers ces nouvelles économies, dès lors qu'elles participent aussi au développement économique local et à la production d'externalités fiscales positives pour les finances des collectivités, à travers l'imposition de ces nouvelles activités dans le cadre d'une fiscalité locale rénovée et, surtout, à travers les impôts locaux que paieraient les particuliers attirés par ce dynamisme. Toutefois, en France, le gouvernement semble a priori préférer la redéfinition d'une assiette commune - qui ne concerne qu'indirectement les collectivités dès lors qu'elles ne sont pas libres de choisir - à travers le remplacement de la fiscalité locale, assise sur l'emprise foncière des magasins, par une taxation fondée sur une assiette commune à tous les acteurs du commerce telles que la CVAE ou la TVA ${ }^{12}$.

\section{B. Un recouvrement de l'impôt " collaboratif " en faveur de l'échelon local}

L'une des principales caractéristiques des économies numériques et collaboratives repose sur la dématérialisation des échanges et de l'ensemble du processus économique. De prime abord, l'intermédiation réalisée par les plateformes limite leur implication économique, leur permettant in fine d'échapper en grande partie à l'impôt dès lors qu'elle ne se soumettent pas aux mêmes obligations réglementaires et fiscales que des entreprises «traditionnelles». Cette problématique est abondamment documentée, ce qui n'est pas forcément le cas de celle qui nous intéresse : il s'agit effectivement de s'interroger sur l'intérêt que les collectivités locales peuvent trouver à cette dématérialisation à travers notamment la modernisation du prélèvement fiscal dont l'administration a aujourd'hui la responsabilité. Contrairement à l'Allemagne par exemple, les collectivités locales ne sont pas elles-mêmes chargées du recouvrement des impôts locaux. II existe des exceptions toutefois éclairantes, notamment à travers la taxe de séjour ${ }^{13}$. Dans les faits, faute d'outils juridiques et moyens matériels adaptés, les communes éprouvent des difficultés à recenser les redevables de la taxe et, par conséquent, à assurer son recouvrement ${ }^{14}$. Si I'hypothèse d'un transfert à l'administration fiscale du recouvrement et du contrôle du paiement de cette taxe a été abordé, en vain, l'idée inverse ne serait-elle pas finalement plus pertinente? Dans le cadre des regroupements de communes - pour une question de rationalisation des coûts -, la création d'une administration fiscale dédiée - ou l'assermentation d'un agent polyvalent -, qui disposerait des moyens de l'administration fiscale d'État avec l'avantage de la mobilité et de la connaissance territoriale, offrirait à n'en pas douter des outils ambitieux au renouvellement et à la clarification de la fiscalité locale. Surtout, elle viendrait décharger de certaines tâches fastidieuses une administration fiscale d'État, sans cesse amenée à davantage dématérialiser ses contrôles; il suffit de penser à la surveillance des réseaux sociaux que l'administration pourra mettre en œuvre à l'avenir ${ }^{15}$. Cette hypothèse suppose toutefois la consécration d'une plus large autonomie fiscale qui, si elle devait être mise en place, devrait l'être à notre avis uniquement dans l'optique d'assujettir à l'impôt les activités que nous pourrions considérer

\footnotetext{
${ }^{10}$ Comité européen des régions, Économie collaborative et plateformes en ligne : points de vue des villes et des régions, $n^{\circ} \mathrm{C} 185$, 9 juin 2017, pp. 24-28.

11 Terrasse Pascal, Rapport au premier ministre sur l'économie collaborative, Rapport février 2016.

${ }^{12}$ Pour un exemple : Jégo Yves, Question écrite n5897, Assemblée nationale, 27 février 2018.

${ }^{13}$ Comité européen des régions, Économie collaborative et plateformes en ligne : points de vue des villes et des régions, ibid.

${ }^{14}$ Commission des finances, de l'économie générale et du contrôle budgétaire, Rapport d'information en conclusion des travaux de la Mission d'évaluation et de contrôle (MEC) sur la fiscalité des hébergements touristiques, Rapport n² 2108, 9 juillet 2014

${ }^{15} \mathrm{~V}$. art. 57 de la loi de finances pour 2020.
} 
o s s i e r

$>$ La fiscalité locale regards comparatifs Colloque de Lyon 10 octobre 2019

${ }^{16}$ Pimont Yves, "Le fisc, le contribuable et les tiers 1). Études de finances publiques: Mélanges en I'honneur du professeur Paul-Marie Gaudemet, Paris, Economica, 1984, p. 641.

${ }^{7}$ Ibid., p. 649.

18 Toutefois, en France, elles pouvaient le faire depuis le 1er janvier 2015 à condition d'en obtenir l'autorisation auprès des collectivités concernées (art. R. 2333-51, (GCT).

${ }^{19}$ Barilari André, "Théorème de la fiscalité locale : la réforme n'est possible que si personne ne perd, sauf l'État! (Cons. const., 14 juin 2013, n²013-323 QPC, Communauté de communes Monts d'Or Azergues)", Constitutions, 2013, p. 433. comme « innovantes » à un certain moment de leur existence, en lieu et place de l'État qui n'est peut-être pas le mieux placé pour intervenir.

Pour l'heure la fiscalité locale est largement dépendante des services de l'État; dès lors, les collectivités ne peuvent atteindre une autonomie suffisante pour réguler par elles-mêmes, et selon leurs propres ambitions, les difficultés spécifiquement posées par les économies numériques. Mais il y a dans la perspective ici présentée une voie de résolution potentielle, à condition de pleinement faire usage des tiers intermédiaires, à savoir les plateformes d'intermédiation - qu'importe d'ailleurs leur nature (dédiées à l'échange ou simples réseaux sociaux). Ces dernières incarnent, pour les collectivités locales, non pas des " auxiliaires passifs $»^{16}$ au prélèvement, dès lors que les informations obtenues nécessiteraient de lourds recoupements et comparaisons ; elles peuvent en revanche incarner des « collaboratrices conscientes ${ }^{17}$ à travers le renseignement et, surtout, le prélèvement à la source qu'elles peuvent mettre en œuvre aisément au profit de l'État et de ses collectivités territoriales. Si l'idée semble glaçante sur le plan des libertés individuelles, une inexorable marche juridique nous en rapproche; en la matière, l'impérieuse nécessité du prélèvement doit dominer avec raison toute conscience collective dont semblent dénués ceux qui ont pour seule « recherche intellectuelle gratifiante » l'évitement de l'impôt. Sur un plan politique, il y aurait sûrement un " prix à payer » à ces plateformes à travers un assouplissement de leurs propres impositions.

Cette prospection prend forme en pratique puisque certaines plateformes numériques, conscientes de l'importance de ne pas susciter I'hostilité des autorités locales, ont rapidement pris parti pour la collaboration avec les municipalités. En ce sens, l'un des exemples les plus aboutis est encore celui de la taxe de séjour : Airbnb avait proposé à plusieurs villes de collecter la taxe et d'en opérer le reversement ${ }^{18}$. Par démarche tout aussi volontaire que mercatique, les plateformes proposaient notamment des pages détaillant les obligations fiscales de leurs utilisateurs. Ainsi, le site Internet d'Airbnb propose aujourd'hui une page « Hébergement responsable en France », dans laquelle il rappelle à ses hôtes l'obligation de respecter la réglementation locale, et fournit quelques informations sommaires concernant ces réglementations et le régime fiscal applicable dans le pays de l'utilisateur. En France, les plateformes sont désormais tenues d'informer l'administration fiscale de l'ensemble des transactions réalisées par l'utilisateur (art. 242 bis, CGI).

Dans le giron du développement des économies numériques et collaboratives - tout aussi implantées sur le territoire que les entreprises traditionnelles -, c'est le tiers collecteur qui semble incarner l'atout le plus précieux des collectivités. Pour autant, il ne convient pas de surestimer le potentiel de cette situation; d'une part car, en parlant de " pathologie de la gouvernance fiscale ${ }^{19}$, André Barilari, rappelle à propos de la fiscalité locale, que le statu quo est préféré à toute innovation - souvent pour des raisons politiques; d'autre part car, si la volonté de l'État est réellement de fournir aux collectivités les moyens de leur financement, alors, soit il convient de leur offrir davantage d'autonomie en matière fiscale - se déchargeant ainsi de toute responsabilité -, soit il convient de leur consentir des dotations plus importantes. Ces prospectives paraissent toutefois temporairement insatisfaisantes à de nombreux égards, l'un des principaux étant que le seul assujettissement des économies numériques et collaboratives aux impôts locaux ne constituerait qu'une part infime des besoins de financement des collectivités. Au fond, si la voie politique choisie n'est pas celle d'une vaste autonomisation fiscale des collectivités, alors sur un plan juridique, c'est celle de la pertinence du maintien d'une fiscalité locale qui doit être interrogée, dès lors que les dotations constituent un système plus efficace à mettre en œuvre - bien que politiquement moins intéressant puisqu'il fait peser la responsabilité politique du financement sur l'État. Si juridiquement des outils peuvent donc être créés, leur portée dépendra essentiellement d'un choix politique. Confrontées à ces pérégrinations, les collectivités n'ont pas fini de pâtir du développement des économies numériques; mais, qu'elles se rassurent : I'État non plus! 